\title{
Estudio de prevalencia de Salmonella sp. en palomas domesticas (Columba livia) en parque zoológico minerva, Quetzaltenango
}

\section{Prevalence study of Salmonella sp. in domestic pigeons (Columba livia) in minerva zoo, Quetzaltenango}

\section{Como citar el artículo}

Amado, V. y Fuentes, H. (2020). Estudio de prevalencia de Salmonella sp. en palomas domesticas (Columba livia) en parque zoológico minerva, Quetzaltenango. Revista Naturaleza, Sociedad y Ambiente, 7 (1), 53-56. DOI: https:// doi.org/10.37533/cunsurori.v7i1.51

Victor Amado Soto y Héctor Fuentes Rousselin

Facultad de Medicina Veterinaria y Zootecnia, Universidad de San Carlos de Guatemala

Recibido: 04 de febrero de 2020 / Aceptado: 28 de agosto de 2020

Disponible en internet el 10 de noviembre de 2020

*Autor para correspondencia, correo electrónico: amadosoto90@gmail.com

\begin{abstract}
Resumen
El estudio consistió en la captura de 30 palomas domésticas, sin importar rango de edad o sexo, situadas en el parque Zoológico Minerva en Quetzaltenango. El objetivo fue determinar la prevalencia de Salmonella sp. en las mismas, a través del método de aglutinación rápida en placa. Investigaciones recientes han reportado que la infección por Salmonella causa 2.8 mil millones de casos de diarrea anualmente en todo el mundo. Salmonella, es considerada la zoonosis con mayor importancia en las enfermedades bacterianas capaz de ser transmitidas por la paloma doméstica. Esta enfermedad ha estado involucrada en morbilidad y mortalidad en aves de zoológicos y de centros de rehabilitación de fauna silvestre en todo el mundo. Sin embargo, no se obtuvieron anticuerpos contra Salmonella $\mathrm{sp}$. en las 30 muestras procesadas, por lo que se concluye que no existe prevalencia de Salmonella sp en palomas domesticas en el zoológico minerva.
\end{abstract}

Palabras clave: Salmonella, Palomas domésticas, Zoológico, Quetzaltenango

\begin{abstract}
The study consisted of capturing 30 domestic pigeons, regardless of age range or sex, located in the Minerva Zoological Park in Quetzaltenango. The objective was to determine the prevalence of Salmonella sp. in them, through the fast plate agglutination method. Recent studies have reported that Salmonella infection causes 2.8 billion cases of diarrhea annually worldwide. Salmonella, is considered the most important zoonosis in bacterial diseases capable of being transmitted by the domestic pigeon. This disease has been involved in morbidity and mortality in birds of zoos and wildlife rehabilitation centers throughout the world. However, no antibodies were found against Salmonella sp. in the 30 samples processed, it concludes that there is no prevalence of Salmonella sp in domestic pigeons in Minerva zoo.
\end{abstract}

Keywords: Salmonella, Domestic pigeons, Zoo, Quetzaltenango 


\section{Introducción}

El género Salmonella pertenece al orden Enterobacteriales y a la familia Enterobacteriaceae que incluye más de 2,300 serotipos. (Herrera, 2015) Investigaciones han estimado que la infección por Salmonella causa 2.8 mil millones de casos de diarrea anualmente en todo el mundo. Se ha informado que Salmonella enterica serovar Typhi (S. Typhi), causa entre 16 y 33 millones de casos infecciosos, con un estimado de 500,000 a 600,000 muertes, mientras que las infecciones por Salmonella no tifoidea (NTS) representan 90 millones de casos y 155000 muertes en todo el mundo anualmente (Abraham, 2018). En los centros de control y prevención de enfermedades se ha estimado que hay aproximadamente 74, 000 casos de salmonelosis asociados a reptiles o anfibios cada año en los Estados Unidos (Bauwens, 2005).

Las aves domésticas y otras especies aviares constituyen el más grande reservorio de Salmonella en la naturaleza, así mismo puede estar presente en animales sanos o enfermos sin el acompañamiento de un cuadro clínico (Herrera. Yonairo, 2015).

Salmonella, es considerada la zoonosis con mayor importancia en las enfermedades bacterianas capaz de ser transmitidas por la paloma doméstica. (González-Acuña, 2007) La mayoría de palomas expuestas a la enfermedad, se recuperan completamente, ya sea mediante tratamiento o a través de mecanismos de defensa naturales (González-Acuña, 2007).

En el parque, Zoológico de Minerva, situado en Quetzaltenango se observa la proliferación de palomas domésticas, las cuales se aprovechan de la comida brindada a los animales mantenidos en cautiverio. Además, las palomas defecan dentro de las jaulas, comederos y/o bebederos, aumentando el riesgo de infección de salmonela a los animales de la colección. El objetivo del estudio fue determinar la prevalencia de Salmonella sp. En palomas domesticas en el parque Zoológico Minerva en la Ciudad de Quetzaltenango, a través del método de aglutinación rápida en placa

\section{Materiales y métodos Área de estudio}

El estudio se realizó en el parque zoológico minerva que se encuentra en la ciudad de Quetzaltenango, localizada a los $14^{\circ} 50^{\prime} 40^{\prime \prime}$ de latitud Norte y $91^{\circ} 30^{\prime} 05^{\prime \prime}$ de longitud oeste, a $206 \mathrm{~km}$ al Noroeste de la ciudad de Guatemala. La ciudad se encuentra ubicada en un valle montañoso en el altiplano occidental con una altitud media sobre el nivel del mar de 2333 metros. Posee tres clases de bosques: latifoliado con 299.72 hectáreas, bosque conífero con 18.88 hectáreas y bosque mixto con 683.38 hectáreas. (Orantes, 2014)

\section{Criterio de inclusión}

Treinta palomas capturadas destinadas a eutanasia como método de control de plagas, sin importar rango edad o sexo.

\section{Técnica de captura}

El parque zoológico minerva realiza controles de plagas (roedores, aves) cada seis meses como una medida para reducir los problemas que las plagas producen. Se utilizaron dos métodos de captura: 1) una canasta de mimbre, un palo de madera, y se colocara alimento para pollos como cebo; y 2) una trampa de red de caída. 


\section{Pesaje}

Se utilizó una balanza electrónica para obtener el peso en gramos de las palomas capturadas.

\section{Toma de muestra}

Se utilizó una jeringa de $3 \mathrm{ml}$ con una aguja 23G para la obtención de sangre de la vena ulnar, humedeciendo el área con alcohol; la sangre se colocó en tubos al vació sin anti coagulante. Luego se separó el suero del coagulo centrifugándolo a 4,000 rpm por cinco minutos, colocándolo en viales de eppendorf para evitar hemolisis.

\section{Técnica de Eutanasia}

Luego de la toma de muestra se administró pentobarbital a dosis de $4 \mathrm{mg}$ por kilo de peso vivo por vía intravenosa en la vena ulnar.

\section{Traslado de la Muestra}

Se enviaron las muestras en una hielera de plástico con hielo seco a una temperatura de cuatro grados centígrados para mantener la cadena de frio, al Laboratorio Regional De Referencia De Sanidad Animal (LARSSA) que se encuentra ubicado en la ciudad de Guatemala.

\section{Procesamiento de la muestra}

Para determinar la presencia de Salmonella sp. en el suero se empleó la prueba de aglutinación rápida en placa.

\section{Resultados y discusión}

En este estudio no se detectó anticuerpos séricos contra Salmonella sp. en ninguna de las 30 muestras de paloma doméstica. Uno de los factores que puede estar asociado en obtener resultados negativos es que la prueba de aglutinación rápida en placa presenta bajos porcentajes de sensibilidad (70.58\%) y especificidad (83.34\%) (Torre-Cisneros, 2010). El fundamento de la prueba se basa en la formación y reconocimiento de complejos antígeno-anticuerpo (Tizard, 2009). Por lo cual si las palomas no han cursado la enfermedad no presentaran anticuerpos contra Salmonella sp.

comportamiento de cada enfermedad en una población depende de múltiples factores, que se han clasificado en tres grandes grupos: factores del propio agente biológico, del medio ambiente y del hospedero. (Marlen Barreto, 2016).

El peso promedio de las palomas domesticas muestreadas fue de 320 gramos, siendo el peso más bajo 210 gramos y el máximo 423 gramos. En la evaluación física presentaban una condición corporal promedio 4 de 9 , según la tabla de índice de condición corporal (BCS). Lo cual puede relacionarse a que tengan un estado inmunitario adecuado y por lo cual no hayan manifestado la enfermedad.

\section{Bibliografía}

Abraham, M. G. T. V. Y. N. D., 2018. Salmonella infection - prevention and treatment by antibiotics and probiotic yeasts: a review. Microbiology Society.

Bauwens, F.-M. S. d. C., 2005. Isolation of salmonella from enviromental samples collected in the reptile department of antwerp zoo using different selective methods, s.l.: Journal of applied microbiology. 
González-Acuña, F. S. G. L. M. S. F. C. L. D. E. J. C. C. y. J. L. M., 2007. Detección de algunos agentes zoonóticos en la paloma(Columbia livia) en la ciudad de Chillán, Chile, Chillan, Chile: Revista chilena de infectologia.

Herrera. Yonairo, M. P. A. S. A. J., 2015. Psitacosis y Salmonelosis: Zoonosis que involucran a las aves. Revista colombiana de Ciencia animal, p. 108.

Herrera, B. Y. J. R. L., 2015. Salmonelosis, zoonosis de las aves y una patogenia muy particular, s.I.: Revista Electronica Veterinaria.
Marlen Barreto, M. C.-R. R., 2016. Salmonella enterica: una revisión de la trilogía agente, hospedero y ambiente, y su trascendencia en Chile, Chile: Infectologia al dia.

Orantes, M. r. m., 2014. Organizacion Empresarial(produccion de maiz), Quetzaltenango: s.n.

Tizard, I. R., 2009. Veterinary Immunology. Missouri: octava edicion.

Torre-Cisneros, R. J. J. C. A. M. A. D. D. R., 2010. Fiebre tifoidea y otras infecciones por salmonella, Cordoba,España: Unidad de Gestión Clínica de Enfermedades Infecciosas.

\section{Sobre autor}

\section{Victor Andrés Amado Soto}

Ejerce como asistente veterinario en el Parque Nacional Zoológico La Aurora, en Guatemala. Estudió la licenciatura de medicina veterinaria en la Universidad de San Carlos de Guatemala (USAC). Realizó su Ejercicio Profesional Supervisado (EPS) en el Parque Zoológico Minerva, en Quetzaltenango, Guatemala. También ha cursado diplomados en línea sobre el manejo, cirugía y medicina interna de animales exóticos y silvestres en el Instituto Mexicano de Fauna Silvestre y Animales de compañía (IMFAC), asimismo realizó prácticas en el Grupo de Rehabilitación Fauna Autóctona y su hábitat (GREFA) en Madrid, España.

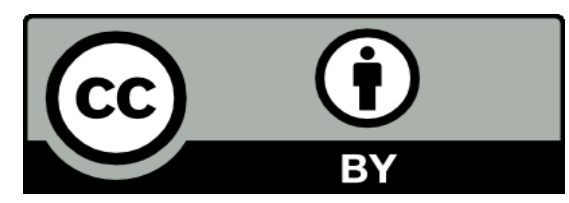

Este texto está protegido por una licencia CreativeCommons 4.0.

Esta licencia permite que otros distribuyan, mezclen, adapten y desarrollen su trabajo, incluso comercialmente, siempre y cuando le den crédito por la creación original. 